Toxicol Appl Pharmacol. 2016 November 15; 311: 34-41. doi:10.1016/j.taap.2016.09.026.

\title{
Role of Dietary Fatty Acids in Liver Injury Caused by Vinyl Chloride Metabolites in Mice
}

\author{
Lisanne C Anders ${ }^{a}, b$, Heegook Yeo ${ }^{a}$, Brenna R Kaelin ${ }^{a}$, Anna L Lang ${ }^{a}$, Adrienne M \\ Bushau $^{\mathrm{a}}$, Amanda N Douglas ${ }^{\mathrm{a}}$, Matt Cave ${ }^{\mathrm{a}, \mathrm{b}, \mathrm{c}, \mathrm{d}, \mathrm{e}}$, Gavin E Arteel $^{\mathrm{a}, \mathrm{c}}$, Craig J McClain ${ }^{\mathrm{a}, \mathrm{b}, \mathrm{c}, \mathrm{d}, \mathrm{e}}$, \\ and Juliane I Beier ${ }^{\mathrm{a}, \mathrm{C}}$ \\ aDepartment of Pharmacology and Toxicology, University of Louisville Health Sciences Center, \\ Louisville, KY 40292, USA \\ bDepartment of Medicine, University of Louisville Health Sciences Center, Louisville, KY 40292, \\ USA \\ 'Hepatobiology and Toxicology Program, University of Louisville Health Sciences Center, \\ Louisville, KY 40292, USA \\ ${ }^{d}$ Diabetes and Obesity Center, University of Louisville Health Sciences Center, Louisville, KY \\ 40292, USA
}

eRobley Rex Louisville VAMC, Louisville, KY 40206

\section{Abstract}

Background-Vinyl chloride (VC) causes toxicant-associated steatohepatitis at high exposure levels. Recent work by this group suggests that underlying liver disease may predispose the liver to VC hepatotoxicity at lower exposure levels. The most common form of underlying liver disease in the developed world is non-alcoholic fatty liver disease (NAFLD). It is well-known that the type of dietary fat can play an important role in the pathogenesis of NAFLD. However, whether the combination of dietary fat and $\mathrm{VC} /$ metabolites promotes liver injury has not been studied.

Methods-Mice were administered chloroethanol (CE - a VC metabolite) or vehicle once, 10 weeks after being fed diets rich in saturated fatty acids (HSFA), rich in poly-unsaturated fatty acids (HPUFA), or the respective low-fat control diets (LSFA; LPUFA).

Results-In control mice, chloroethanol caused no detectable liver injury, as determined by plasma transaminases and histologic indices of damage. In HSFA-fed mice, chloroethanol increased HSFA-induced liver damage, steatosis, infiltrating inflammatory cells, hepatic expression of proinflammatory cytokines, and markers of endoplasmic reticulum (ER) stress. Moreover, markers of inflammasome activation were increased, while markers of inflammasome

Contact information: Juliane I Beier, Ph.D., Department of Pharmacology and Toxicology; University of Louisville; Louisville, KY, 40202, Phone\#: (502) 852-4108; FAX\#: (502) 852-3242; juliane.beier@louisville.edu.

Lisanne C Anders and Heegook Yeo contributed equally to this manuscript.

Publisher's Disclaimer: This is a PDF file of an unedited manuscript that has been accepted for publication. As a service to our customers we are providing this early version of the manuscript. The manuscript will undergo copyediting, typesetting, and review of the resulting proof before it is published in its final citable form. Please note that during the production process errors may be discovered which could affect the content, and all legal disclaimers that apply to the journal pertain. 
inhibition were downregulated. In mice fed HPUFA all of these effects were significantly attenuated.

Conclusions-Chloroethanol promotes inflammatory liver injury caused by dietary fatty acids. This effect is far more exacerbated with saturated fat, versus poly-unsaturated fat; and strongly correlates with a robust activation of the NLRP3 inflammasome in the saturated fed animals only. Taken together these data support the hypothesis that environmental toxicant exposure can exacerbate the severity of NAFLD/NASH.

\section{Keywords}

PVC; TASH; plastic; hepatotoxicity; unsaturated fat; saturated fat

\section{Introduction}

Vinyl chloride is an organochloride used in industry to create the polymer, polyvinyl chloride (PVC), and its production was recently estimated at 27 million metric tons annually (1). VC has been identified as a solvent degradation product at many Superfund sites. It is present in landfill leachate and in the groundwater near military installations where it exists as a microbial metabolite of trichloroethylene (2). Another significant source of exposure is in the chemical industry, especially in the United States, Europe and China (2,3). VC is also found in significant concentrations in the ambient air surrounding manufacturing complexes. However, the main environmental exposure risk stems from contaminated groundwater in areas surrounding production sites. Because $\mathrm{VC}$ is a gas, in homes located above contaminated groundwater, $\mathrm{VC}$ can migrate through soil and foundations to enter basements or living spaces. Owing to its widespread presence in EPA superfund sites and its known potential human risk, VC is ranked \#4 on the ATSDR Hazardous Substance Priority List (4).

$\mathrm{VC}$ is a known human hepatotoxicant that has been demonstrated to cause several benign and malignant diseases, including hepatocellular carcinoma (HCC), hemangiosarcoma and toxicant associated steatohepatitis (5). To date, however, these direct effects of VC exposure are hypothesized to be caused only by high occupational exposures and have limited relevance with existing VC safety regulations. The effect of low environmental VC exposure in contrast to high occupational VC exposures has not been studied. Importantly, the impact of low exposure needs to consider other factors that may increase hepatotoxicity. In this context, the effect of underlying disorders or other insults that may modify risk is critical.

In the liver, the concept of multiple factors contributing to disease is well-known. Indeed, numerous studies have now established that modifications to the liver that are pathologically negligible can become hepatotoxic in response to another factor. This multiple 'hit' paradigm has been described in numerous models of liver injury such as alcoholic $(6,7)$ and non-alcoholic fatty liver diseases $(8,9)$. Although lifestyle and diet are major factors in the etiology of this class of diseases, current research suggests that environmental factors also affect their development and progression. The similarity of $\mathrm{VC}$ metabolism to that of other hepatotoxicants known to sensitize the liver, such as ethanol (10), suggests that $\mathrm{VC}$ may also cause fatty liver. Indeed, recently our laboratory has demonstrated that the VC metabolite chloroethanol renders livers more sensitive to hepatotoxicity caused by the injection of 
bacterial lipopolysaccharide (LPS); this exacerbation of liver damage is characterized by a more robust inflammatory response and enhanced cell death (11).

It has become clear that the type of dietary fat consumed plays an important role in the pathogenesis of liver disease, independent of caloric load (12). For example, in nonalcoholic fatty liver disease (NAFLD), saturated fatty acids (SFA) but not poly-unsaturated fatty acids (PUFA) have been shown to cause inflammation and cell death $(13,14)$. However, it has also been shown that PUFAs can exacerbate liver injury and markers of inflammation in a murine NAFLD model (15). In contrast, in models of alcoholic liver disease (ALD), PUFA (such as linoleic acid) but not SFA, exacerbated alcohol-related liver injury, possibly via pro-inflammatory bioactive oxidized linoleic acid metabolites (OXLAMs) (16-20). Overall, the role of dietary fatty acids and their effects on liver injury and inflammation is still controversial and many aspects remain unclear (12). Importantly, the potential interaction between dietary fat type and $\mathrm{VC} /$ metabolites to promote liver injury has not been studied.

\section{Methods}

\section{Animals and Treatments}

Eighty male C57BL/6J mice, 8 weeks old, from Jackson Laboratory (Bar Harbor, ME) were housed in a pathogen-free barrier facility accredited by the Association for Assessment and Accreditation of Laboratory Animal Care, and procedures were approved by the local Institutional Animal Care and Use Committee. Mice were fed low fat control or high fat diets (see below and Figure 1a and b) for 10 weeks. At the end of the 10 weeks 40 animals were administered chloroethanol (CE; $50 \mathrm{mg} / \mathrm{kg}$ i.g.) and 40 animals were administered vehicle (water) 24 hours prior to sacrifice. The concentration of $\mathrm{CE}$ was determined by others not to directly cause liver damage (21) and was validated in a previously published study by our group (11). Based on the fraction of VC that is estimated to be metabolized to $\mathrm{CE}$ and its apparent volume of distribution in rodents, this concentration equates to $\sim 100$ ppm inhalation exposure of VC over a short period of time. At sacrifice, animals were anesthetized with ketamine/xylazine (100/15 mg/kg, i.m.). Blood was collected from the vena cava just prior to sacrifice (exsanguination), and citrated plasma was stored at $-80^{\circ} \mathrm{C}$ for further analysis. Portions of liver tissue were snap-frozen in liquid nitrogen, embedded in frozen specimen medium (Sakura Finetek, Torrance, CA), or were fixed in 10\% neutral buffered formalin.

Low saturated fat diet (LSFA)—[13\% calories as fat; Casein $195.0 \mathrm{~g} / \mathrm{kg}$, DLMethionine $3.0 \mathrm{~g} / \mathrm{kg}$, Sucrose $120.0 \mathrm{~g} / \mathrm{kg}$, Corn Starch $432.89 \mathrm{~g} / \mathrm{kg}$, Maltodextrin $100.0 \mathrm{~g} / \mathrm{kg}$, Anhydrous Milkfat $37.2 \mathrm{~g} / \mathrm{kg}$, Soybean Oil $12.8 \mathrm{~g} / \mathrm{kg}$, Cellulose $50.0 \mathrm{~g} / \mathrm{kg}$, Mineral Mix, AIN-76 (170915) 35.0 g/kg, Calcium Carbonate 4.0 g/kg, Vitamin Mix, Teklad (40060) 10.0 $\mathrm{g} / \mathrm{kg}$, Ethoxyquin, antioxidant $0.01 \mathrm{~g} / \mathrm{kg}$; (Harlan Laboratories, Madison, WI)].

High saturated fat diet (HSFA)—[42\% calories as fat; Casein $195.0 \mathrm{~g} / \mathrm{kg}$, DLMethionine $3.0 \mathrm{~g} / \mathrm{kg}$, Sucrose $341.31 \mathrm{~g} / \mathrm{kg}$, Corn Starch $75.0 \mathrm{~g} / \mathrm{kg}$, Maltodextrin $75.0 \mathrm{~g} / \mathrm{kg}$, Anhydrous Milkfat $210.0 \mathrm{~g} / \mathrm{kg}$, Cholesterol $1.5 \mathrm{~g} / \mathrm{kg}$, Cellulose $50.0 \mathrm{~g} / \mathrm{kg}$, Mineral Mix, 
AIN-76 (170915) 35.0 g/kg, Calcium Carbonate 4.0 g/kg, Vitamin Mix, Teklad (40060) 10.0 $\mathrm{g} / \mathrm{kg}$, Ethoxyquin, antioxidant $0.04 \mathrm{~g} / \mathrm{kg}$; (Harlan Laboratories, Madison, WI)].

Low polyunsaturated fat diet (LPUFA)—[13\% calories as fat; Casein $195.0 \mathrm{~g} / \mathrm{kg}$, DLMethionine $3.0 \mathrm{~g} / \mathrm{kg}$, Sucrose $120.0 \mathrm{~g} / \mathrm{kg}$, Corn Starch $432.79 \mathrm{~g} / \mathrm{kg}$, Maltodextrin $100.0 \mathrm{~g} / \mathrm{kg}$, Corn Oil $50.0 \mathrm{~g} / \mathrm{kg}$, Cholesterol $0.1 \mathrm{~g} / \mathrm{kg}$, Cellulose $50.0 \mathrm{~g} / \mathrm{kg}$, Mineral Mix, AIN-76 (170915) $35.0 \mathrm{~g} / \mathrm{kg}$, Calcium Carbonate $4.0 \mathrm{~g} / \mathrm{kg}$, Vitamin Mix, Teklad (40060) $10.0 \mathrm{~g} / \mathrm{kg}$, Ethoxyquin, antioxidant $0.01 \mathrm{~g} / \mathrm{kg}$; (Harlan Laboratories, Madison, WI)]

High polyunsaturated fat diet (HPUFA)—[42\% calories as fat; Casein $195.0 \mathrm{~g} / \mathrm{kg}$, DLMethionine $3.0 \mathrm{~g} / \mathrm{kg}$, Sucrose $341.36 \mathrm{~g} / \mathrm{kg}$, Corn Starch $49.5 \mathrm{~g} / \mathrm{kg}$, Maltodextrin $100.0 \mathrm{~g} / \mathrm{kg}$, Corn Oil 210.0 g/kg, Cholesterol 2.0 g/kg, Cellulose 50.0 g/kg, Mineral Mix, AIN-76 (170915) $35.0 \mathrm{~g} / \mathrm{kg}$, Calcium Carbonate $4.0 \mathrm{~g} / \mathrm{kg}$, Vitamin Mix, Teklad (40060) $10.0 \mathrm{~g} / \mathrm{kg}$, Ethoxyquin, antioxidant $0.04 \mathrm{~g} / \mathrm{kg}$; (Harlan Laboratories, Madison, WI)]

\section{Biochemical Analyses, Histology and Immunohistochemistry}

Oral glucose tolerance was evaluated at 4 and 8 weeks during the feeding protocol (Figure 1a for timeline). Mice were fasted for 6 hours, then blood was sampled via tail cut immediately after fasting to determine baseline. Following oral administration of $2 \mathrm{mg} / \mathrm{kg}$ D-/(+)-glucose (Sigma, St. Louis, MO) in $4 \mathrm{ml} / \mathrm{kg}$ of sterile saline solution, blood was sampled and glucose concentrations measured at 15, 30, 60 and 90 minutes. Glucose concentrations were determined using an Accu-Chek Aviva Plus glucometer and test strips (Roche Diagnostics Corp., Indianapolis, IN). Plasma transaminases (ALT and AST) were determined using standard kits (Thermo Fisher Scientific, Middletown, VA). Paraffin embedded liver sections were stained with hematoxylin \& eosin (H\&E) and neutrophil accumulation was assessed by chloroacetate esterase stain (CAE; Sigma, St. Louis MO). CAE-positive cells were counted using Metamorph Image Analysis Software (Molecular Devices, Sunnyvale, CA) and are expressed as positive cells per 1000 hepatocytes. Hepatic lipids were extracted from snap-frozen liver samples as described previously $(22,23)$. Hepatic and plasma lipids were determined using standard clinical chemistry reagents for cholesterol, and triglycerides (Infinity, Thermo Fisher Scientific, Middletown, VA). Liver sections were stained with Oil Red O (ORO) for visualization of neutral lipids, as described previously (7).

\section{Immunoblots}

Liver samples were homogenized in RIPA buffer (24) containing protease and phosphatase inhibitor cocktails (Sigma, St. Louis, MO). Samples were loaded onto SDS-polyacrylamide gels (Invitrogen, Thermo Fisher Scientific, Grand Island, NY), followed by electrophoresis and Western blotting onto PVDF membranes (Hybond P, GE Healthcare Bio-Sciences, Pittsburgh, PA). Primary polyclonal antibodies for mouse ATF3, CHOP, HMGB1, Caspase 1, and NLRP3 were used and compared to GAPDH (Cell Signaling Technology; Beverly, MA). Densitometric analysis was performed using UN-SCAN-IT gel (Silk Scientific Inc., Orem, UT) software. 


\section{RNA Isolation and Real-Time RT-PCR}

RNA was extracted immediately following sacrifice from fresh liver samples using RNA Stat60 and chloroform. Real-time RT-PCR was performed using a StepOne real time PCR system (Thermo Fisher Scientific, Grand Island, NY). Primers and probes were ordered as commercially available kits (Thermo Fisher Scientific, Grand Island, NY). The comparative $\mathrm{C}_{\mathrm{T}}$ method was used to determine fold differences between the target genes and an endogenous reference (18S).

\section{Statistical Analyses}

Results are reported as means \pm SEM $(n=4-7)$ and were analyzed using SigmaPlot 11.0 (Systat Software, Inc.; San Jose, CA). ANOVA with Bonferroni's post-hoc test (for parametric data) or Mann-Whitney Rank Sum test (for nonparametric data) were used for the determination of statistical significance among treatment groups, as appropriate. Student's t-test and Fisher's exact test were used to determine statistical significance between human subjects groups. A p value $<0.05$ was selected before the study as the level of significance.

\section{Results}

\section{High fat diet causes metabolic syndrome}

Figure 1c shows the results of oral glucose tolerance testing (OGTT). Blood glucose levels of both high fat diet groups were increased significantly over their respective control diets. While blood glucose levels of HPUFA-fed animals were higher at 15 and 30 minutes when compared with HSFA-fed animals, cumulative blood glucose levels, however were not different (Figure 1c inset). Figure 1d shows the amount of food consumed by each dietary group over the 10-week period. All dietary groups consumed comparable amounts of food throughout the feeding period.

\section{Effect of CE on liver injury and steatosis caused by high fat diet}

Figure 2 shows representative photomicrographs depicting liver pathology (H\&E, Figure 2a) and neutrophil accumulation (CAE, Figure 2b) 24 hours after CE. No pathological changes were observed in the two low fat diet control groups (Figure 2a; H\&E) and this effect was unchanged by CE. Compared with the low fat diet groups, there was a significant increase in neutrophil recruitment in both the HPUFA and the HSFA group. Interestingly, CE significantly enhanced this effect after HSFA feeding, while there were no differences observed in the HPUFA group (Figures $2 b$ and $2 d$ ). In line with these results, plasma levels of transaminases were within normal range in mice fed a low fat diet (Figure 2c) and CE did not significantly alter this effect. In the HSFA group however, plasma transaminases were significantly elevated, which was further increased by CE. While HPUFA-feeding alone caused no increase in transaminase levels, CE significantly increased both ALT and AST levels, albeit less robustly than with HSFA-feeding (Figure 2c).

Circulating and hepatic lipid concentrations were measured (Figure 3a and b). CE did not affect the circulating and hepatic concentrations of triglycerides and cholesterol in mice fed low fat diets (Figure 3a and b). Circulating triglyceride levels were increased in the high fat 
diet groups only when CE was present. While high saturated fatty acids alone increased circulating cholesterol levels, this effect was increased by CE. High poly-unsaturated fatty acids diets alone caused no changes in circulating cholesterol levels but caused an elevation in combination with CE (Figure 3a). While hepatic triglyceride and cholesterol levels were increased with both high fat diets when compared to their respective low fat controls, CE significantly increased triglyceride levels in both high fat diet groups. However this effect was much less robust in the HPUFA group. Interestingly, CE increased cholesterol levels in the HSFA group but attenuated them in the HPUFA group (Figure 3b). In line with hepatic lipid level results, hepatic expression of lipogenic factor Fsp27 (fat-specific protein 27, Cidec--involved in lipid droplet formation) was increased in both high fat diets (Figure 3c). While CE further increased this effect in both diets, the effect was less prominent in the HPUFA diet. The changes in hepatic TG levels and hepatic Fsp27 expression were also reflected in similar increases in lipid droplets in livers of mice, as determined by ORO stains (Figure 3d).

\section{Changes in markers of ER stress in response to an interaction of high fat diet and CE}

Previous studies have shown that steatosis and hepatic lipid droplet formation can be caused by ER stress. Indeed, Fsp27 has been shown to be inducible by ER stress (25). Therefore, changes in hepatic mRNA expression and protein for markers of ER stress were determined (Figure 4). Hepatic mRNA expression of key markers of ER stress (Atf3 and Chop) was unaffected by $\mathrm{CE}$ in the control groups. Moreover, expression was also unaffected by either of the HFDs. However, CE significantly increased both Atf 3 and Chop in the HSFA group. While CE also increased Chop expression in the HPUFA group, the effect was significantly attenuated when compared with the combination of CE and HSFA (Figure 4a).

\section{Effect of CE on the induction of inflammatory gene expression caused by high fat diet}

As CE enhances recruitment of inflammatory cells caused by fatty diet (Figures $2 b$ and $2 c$ ), its effect on hepatic expression of key inflammatory genes involved in the M1 response (Tnfa, Pai-1 and II-1 $\beta$ ) was determined (Figures $4 \mathrm{~b}$ and $4 \mathrm{c}$ ). CE did not significantly change the expression for any of the genes in the control groups. While expression of both Pai-1 and II- $1 \beta$ was significantly increased by both high fat diets, expression of Tnfa was unchanged. The combination of HSFA and CE significantly enhanced expression for all of these markers. However, CE caused no changes in the expression of Tnfa, only slightly increased the expression of Pai- 1 and significantly attenuated $I I-1 \beta$ expression in the HPUFA group (Figures $4 \mathrm{~b}$ and $4 \mathrm{c}$ ). In line with the II- $1 \beta$ mRNA expression data (Figure $4 \mathrm{~B}$ upper right panel), plasma levels of IL-1 $\beta$ protein in HSFA, as determined by ELISA, were strongly increased with CE, while CE attenuated IL-1 $\beta$ plasma protein concentration in the HPUFA group (Figure $4 \mathrm{c}$ lower right panel).

\section{Effects of chloroethanol on inflammasome activation}

Recent studies have indicated that inflammasome activation is critical in NAFLD initiation and progression $(13,14)$. It has been shown that, while some dietary fatty acids contribute to inflammasome activation, others do not $(26,27)$. Moreover, here we show that both hepatic II- $1 \beta$ expression and plasma levels of IL-1 $\beta$ protein, a key marker of inflammasome activation, were elevated in animals fed HSFA plus CE, and this effect was blunted in 
animals fed HPUFA. Therefore, additional markers of inflammasome activation were analyzed. Expression of genes involved in inflammasome activation (NIrp3, NIrC4, and Asc; Figure 5a) and inhibition of inflammasome activation (Sirt1, and Ppary, Figure 5b) are shown. While expression of none of these markers was changed in the control groups, CE increased Nlrp3, Nlrc4, and Asc, expression caused by HSFA Figure 5a). However, in the HPUFA group, CE significantly attenuated this effect. Expression of Sirt1 and Ppar $\gamma$, associated with inflammasome suppression was significantly decreased by HSFA+CE. Expression Sirt1 of but not of Ppar $\gamma$ was increased by HPUFA+CE (Figure 5b). Proteins associated with inflammasome activation were measured via western blot analysis (Figure 6). Hepatic protein expression of Caspase1, and NLRC3, which are components of the assembled inflammasome, as well as HMGB1 (a damage-associated molecular pattern-DAMP) were determined via Western Blot analysis (Figure 6). In line with plasma IL-1 $\beta$ concentrations (Figure 4c), hepatic protein expression of these markers and caspase 1 cleavage was increased in the HSFA+CE group (Figure 6). While HMGB1 was also increased by HPUFA+CE, NLRP3 protein and caspase 1 cleavage was not.

\section{Discussion}

A major paradigm shift in environmental research in the past years has been to investigate the impact of moderate/low exposures in contrast to high/occupational exposures. This 'exposure biology' approach attempts to take into account multiple aspects that may contribute to the final phenotype of risk. In this context, the impact of underlying disorders that may modify risk is critical. The pandemic of obesity is arguably the most prevalent underlying disorder that may impact the US population. Obesity is a primary risk factor for a myriad of health complications, including diabetes, cancer and cardiovascular disease. Nonalcoholic fatty liver disease (NAFLD), which is closely associated with metabolic syndrome, has increased in the United States in parallel with the obesity epidemic (28). However, not all obese individuals develop clinically-relevant liver disease directly linked to their obesity. It is clear that the risk for developing liver disease is not based solely on obesity, but is rather modified by other mitigating conditions, such as other environmental or genetic factors. Furthermore, the impact of obesity on the risk of developing diseases caused by other events, such as environmental exposure, is poorly understood at this time.

As mentioned in the Introduction the ubiquity and potential for harm places $\mathrm{VC}$ exposure high on the list of environmental health concerns (2). The direct hepatotoxicity of VC exposure has been established, but requires relatively high occupational exposure. Current safety restrictions in today's industry lessen the risks of high exposures, but exposures to lower environmental concentrations that are not overtly hepatotoxic persist. In contrast, little is known about the potential impact of these exposure levels on hepatic health, especially in the context of the ever increasing burden of underlying liver disease. We have previously shown in a proof-of-concept study that the $\mathrm{VC}$ metabolite $\mathrm{CE}$ sensitizes the liver to a second insult, LPS (11). Although elevated portal and/or systemic LPS is common in human diseases, high acute endotoxemia, such as modeled in that study (11), is a rare event. The results summarized herein support the hypothesis that chloroethanol exposure enhances experimental NAFLD in mice fed a high fat diet. In toto, these studies suggest that experimental VC exposures that are subhepatotoxic may cause liver injury when combined 
with other factors. These results furthermore add weight to the hypothesis that environmental exposure to $\mathrm{VC}$ may exacerbate underlying liver disease.

Risk factors for NAFLD are analogous to those of metabolic syndrome (e.g., obesity, type II diabetes, and dyslipidemia) (29). As with other sequelae of metabolic syndrome (e.g., cardiovascular diseases), the type of fat, in addition to the amount, appears to be critical in mediating risk. Specifically, in human studies and in experimental models, saturated fats appear to increase the risk of developing NAFLD/NASH in contrast to diets enriched in unsaturated fats (30). However, the role of unsaturated fat remains controversial and may not unilaterally be categorized as the less detrimental fat source (12). Indeed in contrast, saturated fats appear to protect against liver disease caused by alcohol (i.e., alcoholic liver disease; ALD) (31-33). The chemical structure and metabolism of CE, the primary VC metabolite, is similar to that of ethanol. It was therefore unclear a priori what the impact of the type of dietary fat may be on VC metabolite exposure. Thus, an additional goal of this project was therefore to investigate the impact of dietary fat type on this interaction. We observed that the enhancement of HFD-induced liver damage by CE exposure was more robust in animals fed SFAs than in animals fed PUFAs (Figures 2-4). All aspects of liver pathology in this model (e.g., steatosis, inflammation and cell death) appeared to be increased by the combination of diet and CE exposure (Figures 2-4), suggesting that this interaction was via a mechanism central to injury.

It has been shown that the liver participates in the metabolic derangements of the metabolic syndrome and that hepatic steatosis plays a major role in this process $(34,35)$. Fatty livers are also more sensitive to injury caused by an additional hepatotoxicant $(8,9)$. Previous studies have indicated that the degree of the fat accumulation varies depending on the dietary fat content. SFAs have been shown to promote steatosis more than PUFAs (36). Thus, differences in lipid accumulation in response to saturated versus unsaturated fat diets could contribute to the observed increase in sensitivity. With our results, we confirm the work of others showing that SFAs cause more robust hepatic steatosis than PUFAs (Figure 3) (30). Although CE exposure increased steatosis and inflammation in livers from mice fed high-fat diets, the effect was much more pronounced in the SFA group. Taken together, these data indicate that a diet high in saturated fats makes the liver more sensitive to the hepatotoxicity of CE.

Multiple factors are involved during the progression of NAFLD from steatosis to steatohepatitis, and progressive liver damage. Recent work has suggested that inflammasome activation is a central mechanism in this progression $(13,37,38)$. Further, it has been shown that dietary saturated fatty acids are more potent at activating the inflammasome in experimental NAFLD compared to unsaturated fatty acids $(14,26,27)$. Here we indeed observed that the enhancement of HFD induced liver damage by CE correlated with a concomitant increase in inflammasome activation in the HSFA group (Figures 5 and 6). The canonical activators of the inflammasome are pathogen associated molecular patterns (PAMPs; e.g., LPS) or damage-associated molecular patterns (DAMPs; e.g., HMGB1). DAMPs, such as reactive oxygen species (ROS) or products of necrosis (e.g., ATP) may directly activate the inflammasome $(39,40)$. Previously, we demonstrated that VCmetabolite induced cell death favors necrosis (11), which supports this mechanism of action. 
Lipid-derived products (e.g., free fatty acids and cholesterol) themselves are endogenous DAMPs, and directly activate the inflammasome via both TLR and NLR pathways $(13,14)$. A recent study also demonstrates that ER stress also enhances inflammasome activity through CHOP (41). All of these DAMP-producing responses were elevated in the HSFA + CE group. Moreover, hepatic expression of inhibitors of inflammasome activation, Ppary and Sirt1 (42, 43), were blunted in HSFA+CE. In summary, several converging mechanisms likely contribute to the enhanced hepatic inflammasome activation caused by HSFA diet when mice are exposed to CE.

\section{Summary and Conclusions}

The results of this study suggest that the VC metabolite, chloroethanol, causes increased inflammation, increased inflammasome activation and oxidative ER stress when combined with a diet enriched in saturated fatty acids, while a diet enriched in PUFA had much less robust effects. These results suggest that saturated fatty acids sensitize the liver to CE much more strongly than unsaturated fatty acids. Although not specifically addressed in the current study, these data raise concerns about potential for overlap between diets high in saturated fats (i.e. Western diet) and exposure to VC (and metabolites); namely, both factors tend to be higher in areas of lower socioeconomic status (44-46).

\section{Acknowledgments}

Funding: This study was funded by awards from the National Institutes of Health: K01 DK096042, R03 DK107912 to Juliane Beier; T32ES011564 to Gavin Arteel and R01AA023681, T35ES014559, U01AA021893, U01AA021901, to Craig J McClain.

Research was also supported by an Institutional Development Award (IDeA) from the National Institute of General Medical Sciences of the National Institutes of Health under grant number P20GM113226 and the National Institute on Alcohol Abuse and Alcoholism of the National Institutes of Health under Award Number P50AA024337. The content is solely the responsibility of the authors and does not necessarily represent the official views of the National Institutes of Health.

\section{Reference List}

1. Sass JB, Castleman B, Wallinga D. Vinyl chloride: a case study of data suppression and misrepresentation. Environ Health Perspect. 2005 Jul; 113(7):809-812. [PubMed: 16002366]

2. Kielhorn J, Melber C, Wahnschaffe U, Aitio A, Mangelsdorf I. Vinyl chloride: still a cause for concern. Environ Health Perspect. 2000 Jul; 108(7):579-588. [PubMed: 10905993]

3. WHO. Vinyl Chloride in Drinking-water. 2004 Report No.: WHO/SDE/WSH/03.04/119.

4. U.S.Department of Health and Human Services PHS. Agency for Toxic Substances and Disease Registry (ATSDR). Toxicological profile for Vinyl Chloride. 2006

5. Wahlang B, Beier JI, Clair HB, Bellis-Jones HJ, Falkner KC, McClain CJ, et al. Toxicant-associated steatohepatitis. Toxicol Pathol. 2013 Feb; 41(2):343-360. [PubMed: 23262638]

6. Beier JI, Arteel GE, McClain CJ. Advances in alcoholic liver disease. Curr Gastroenterol Rep. 2011 Feb; 13(1):56-64. [PubMed: 21088999]

7. Beier JI, Luyendyk JP, Guo L, von Montfort C, Staunton DE, Arteel GE. Fibrin accumulation plays a critical role in the sensitization to lipopolysaccharide-induced liver injury caused by ethanol in mice. Hepatology. 2009 Jan 23; 49(5):1545-1553. [PubMed: 19291788]

8. Yang SQ, Lin HZ, Lane MD, Clemens M, Diehl AM. Obesity increases sensitivity to endotoxin liver injury: implications for the pathogenesis of steatohepatitis. Proc Natl Acad Sci U S A. 1997; 94:2557-2562. [PubMed: 9122234] 
9. Day CP, James OF. Steatohepatitis: a tale of two "hits"? Gastroenterology. 1998 Apr; 114(4):842845. [PubMed: 9547102]

10. Bolt HM. Vinyl chloride-a classical industrial toxicant of new interest. Crit Rev Toxicol. 2005 Apr; 35(4):307-323. [PubMed: 15989139]

11. Anders LC, Lang AL, Anwar-Mohamed A, Douglas AN, Bushau AM, Falkner KC, et al. Vinyl Chloride Metabolites Potentiate Inflammatory Liver Injury Caused by LPS in Mice. Toxicol Sci. 2016 Jun; 151(2):312-323. [PubMed: 26962056]

12. Juarez-Hernandez E, Chavez-Tapia NC, Uribe M, Barbero-Becerra VJ. Role of bioactive fatty acids in nonalcoholic fatty liver disease. Nutr J. 2016; 15(1):72. [PubMed: 27485440]

13. Csak T, Ganz M, Pespisa J, Kodys K, Dolganiuc A, Szabo G. Fatty acid and endotoxin activate inflammasomes in mouse hepatocytes that release danger signals to stimulate immune cells. Hepatology. 2011 Jul; 54(1):133-144. [PubMed: 21488066]

14. Miura K, Yang L, van RN, Brenner DA, Ohnishi H, Seki E. Toll-like receptor 2 and palmitic acid cooperatively contribute to the development of nonalcoholic steatohepatitis through inflammasome activation in mice. Hepatology. 2013 Feb; 57(2):577-589. [PubMed: 22987396]

15. Provenzano A, Milani S, Vizzutti F, Delogu W, Navari N, Novo E, et al. n-3 polyunsaturated fatty acids worsen inflammation and fibrosis in experimental nonalcoholic steatohepatitis. Liver Int. 2014 Jul; 34(6):918-930. [PubMed: 24628836]

16. Nanji AA, Sadrzadeh SM, Yang EK, Fogt F, Meydani M, Dannenberg AJ. Dietary saturated fatty acids: a novel treatment for alcoholic liver disease. Gastroenterology. 1995; 109(2):547-554. [PubMed: 7615205]

17. Kirpich IA, Feng W, Wang Y, Liu Y, Beier JI, Arteel GE, et al. Ethanol and dietary unsaturated fat (corn oil/linoleic acid enriched) cause intestinal inflammation and impaired intestinal barrier defense in mice chronically fed alcohol. Alcohol. 2013 May; 47(3):257-264. [PubMed: 23453163]

18. Martinez-Clemente M, Ferre N, Titos E, Horrillo R, Gonzalez-Periz A, Moran-Salvador E, et al. Disruption of the 12/15-lipoxygenase gene (Alox15) protects hyperlipidemic mice from nonalcoholic fatty liver disease. Hepatology. 2010 Dec; 52(6):1980-1991. [PubMed: 20967760]

19. Uderhardt S, Kronke G. 12/15-lipoxygenase during the regulation of inflammation, immunity, and self-tolerance. J Mol Med (Berl). 2012 Nov; 90(11):1247-1256. [PubMed: 22983484]

20. Vangaveti V, Baune BT, Kennedy RL. Hydroxyoctadecadienoic acids: novel regulators of macrophage differentiation and atherogenesis. Ther Adv Endocrinol Metab. 2010 Apr; 1(2):51-60. [PubMed: 23148150]

21. Kaphalia BS, Ansari GA. Hepatic fatty acid conjugation of 2-chloroethanol and 2-bromoethanol in rats. J Biochem Toxicol. 1989; 4(3):183-188. [PubMed: 2607542]

22. Bligh EG, Dyer WJ. A rapid method of total lipid extraction and purification. Can J Biochem Physiol. 1959; 37:911-917. [PubMed: 13671378]

23. Kaiser JP, Beier JI, Zhang J, David HJ, von Montfort C, Guo L, et al. PKCepsilon plays a causal role in acute ethanol-induced steatosis. Arch Biochem Biophys. 2009 Feb; 482(1-2):104-111. [PubMed: 19022218]

24. Beier JI, von MC, Sies H, Klotz LO. Activation of ErbB2 by 2-methyl-1,4-naphthoquinone (menadione) in human keratinocytes: role of EGFR and protein tyrosine phosphatases. FEBS Lett. 2006 Mar 20; 580(7):1859-1864. [PubMed: 16516204]

25. Lee JS, Mendez R, Heng HH, Yang ZQ, Zhang K. Pharmacological ER stress promotes hepatic lipogenesis and lipid droplet formation. Am J Transl Res. 2012; 4(1):102-113. [PubMed: 22347525]

26. Sui YH, Luo WJ, Xu QY, Hua J. Dietary saturated fatty acid and polyunsaturated fatty acid oppositely affect hepatic NOD-like receptor protein 3 inflammasome through regulating nuclear factor-kappa B activation. World J Gastroenterol. 2016 Feb 28; 22(8):2533-2544. [PubMed: 26937141]

27. Camell C, Goldberg E, Dixit VD. Regulation of Nlrp3 inflammasome by dietary metabolites. Semin Immunol. 2015 Sep; 27(5):334-342. [PubMed: 26776831]

28. Cave M, Deaciuc I, Mendez C, Song Z, Joshi-Barve S, Barve S, et al. Nonalcoholic fatty liver disease: predisposing factors and the role of nutrition. J Nutr Biochem. 2007 Mar; 18(3):184-195. [PubMed: 17296492] 
29. Clark JM. The epidemiology of nonalcoholic fatty liver disease in adults. J Clin Gastroenterol. 2006 Mar; 40(3 Suppl 1):S5-10. [PubMed: 16540768]

30. Gentile CL, Pagliassotti MJ. The role of fatty acids in the development and progression of nonalcoholic fatty liver disease. J Nutr Biochem. 2008 Sep; 19(9):567-576. [PubMed: 18430557]

31. Nanji AA, Mendenhall CL, French SW. Beef fat prevents alcoholic liver disease in the rat. Alcohol Clin Exp Res. 1989; 13:15-19. [PubMed: 2646971]

32. Nanji AA, Yang EK, Fogt F, Sadrzadeh SM, Dannenberg AJ. Medium chain triglycerides and vitamin $E$ reduce the severity of established experimental alcoholic liver disease. J Pharmacol Exp Ther. 1996 Jun; 277(3):1694-1700. [PubMed: 8667240]

33. Nanji AA, Jokelainen K, Tipoe GL, Rahemtulla A, Dannenberg AJ. Dietary saturated fatty acids reverse inflammatory and fibrotic changes in rat liver despite continued ethanol administration. J Pharmacol Exp Ther. 2001 Nov; 299(2):638-644. [PubMed: 11602676]

34. den Boer M, Voshol PJ, Kuipers F, Havekes LM, Romijn JA. Hepatic steatosis: a mediator of the metabolic syndrome. Lessons from animal models. Arterioscler Thromb Vasc Biol. 2004 Apr; 24(4):644-649. [PubMed: 14715643]

35. Lonardo A, Ballestri S, Marchesini G, Angulo P, Loria P. Nonalcoholic fatty liver disease: a precursor of the metabolic syndrome. Dig Liver Dis. 2015 Mar; 47(3):181-190. [PubMed: 25739820]

36. Ferramosca A, Zara V. Modulation of hepatic steatosis by dietary fatty acids. World J Gastroenterol. 2014 Feb 21; 20(7):1746-1755. [PubMed: 24587652]

37. Petrasek J, Bala S, Csak T, Lippai D, Kodys K, Menashy V, et al. IL-1 receptor antagonist ameliorates inflammasome-dependent alcoholic steatohepatitis in mice. J Clin Invest. 2012 Oct 1; 122(10):3476-3489. [PubMed: 22945633]

38. Wree A, McGeough MD, Pena CA, Schlattjan M, Li H, Inzaugarat ME, et al. NLRP3 inflammasome activation is required for fibrosis development in NAFLD. J Mol Med (Berl). 2014 Oct; 92(10):1069-1082. [PubMed: 24861026]

39. Elinav E, Henao-Mejia J, Flavell RA. Integrative inflammasome activity in the regulation of intestinal mucosal immune responses. Mucosal Immunol. 2013 Jan; 6(1):4-13. [PubMed: 23212196]

40. Schroder K, Tschopp J. The inflammasomes. Cell. 2010 Mar 19; 140(6):821-832. [PubMed: 20303873]

41. Lebeaupin C, Proics E, de Bieville CH, Rousseau D, Bonnafous S, Patouraux S, et al. ER stress induces NLRP3 inflammasome activation and hepatocyte death. Cell Death Dis. 2015; 6:e1879. [PubMed: 26355342]

42. Li Y, Xu S, Jiang B, Cohen RA, Zang M. Activation of sterol regulatory element binding protein and NLRP3 inflammasome in atherosclerotic lesion development in diabetic pigs. PLoS One. 2013; 8(6):e67532. [PubMed: 23825667]

43. Collino M, Benetti E, Rogazzo M, Mastrocola R, Yaqoob MM, Aragno M, et al. Reversal of the deleterious effects of chronic dietary HFCS-55 intake by PPAR-delta agonism correlates with impaired NLRP3 inflammasome activation. Biochem Pharmacol. 2013 Jan 15; 85(2):257-264. [PubMed: 23103566]

44. James WP, Nelson M, Ralph A, Leather S. Socioeconomic determinants of health. The contribution of nutrition to inequalities in health. BMJ. 1997 May 24; 314(7093):1545-1549. [PubMed: 9183207]

45. Laplanche A, Clavel F, Contassot JC, Lanouziere C. Exposure to vinyl chloride monomer: report on a cohort study. Br J Ind Med. 1987 Oct; 44(10):711-715. [PubMed: 3676124]

46. Hu J, Mao Y, White K. Renal cell carcinoma and occupational exposure to chemicals in Canada. Occup Med (Lond). 2002 May; 52(3):157-164. [PubMed: 12063361] 


\section{Highlights}

- $\quad$ CE promotes inflammatory liver injury caused by dietary fatty acids

- $\quad$ This effect is stronger with saturated than with unsaturated fatty acids

- $\quad$ Damage caused by saturated fat and CE correlates with inflammasome activation 
a

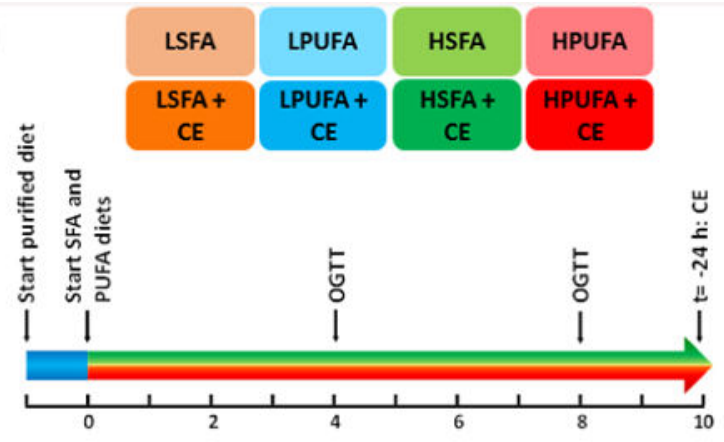

b

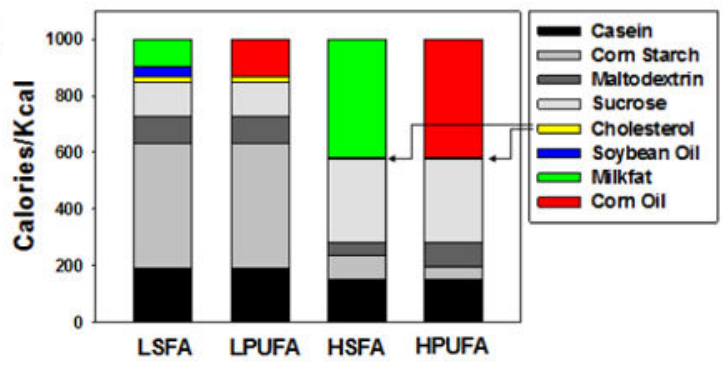

C
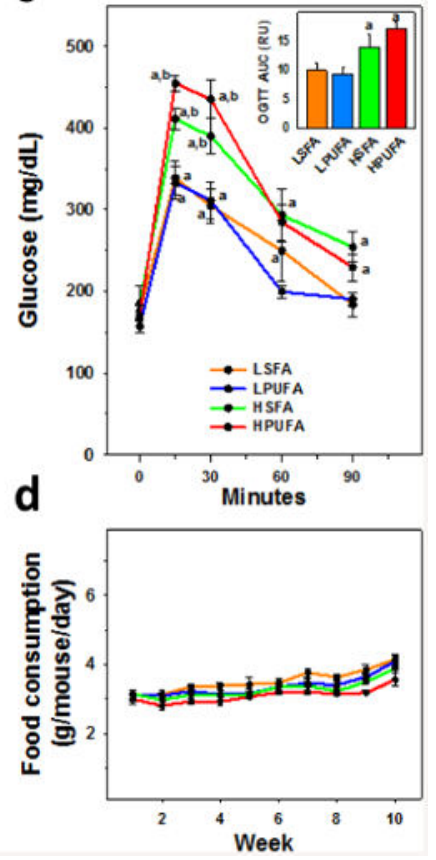

Figure 1. The experimental animal model of non-alcoholic liver disease and its effect on glucose tolerance in mice

a: Schematic presentation of the feeding protocol and animal experiment with 10 animals per group, as described in Materials and Methods. b: Composition of the experimental diets. The SFA diets were enriched with milkfat. The PUFA diets were enriched with corn oil. Soybean oil was used to provide essential free fatty acids in the low milkfat diet (LSFA). The control (LSFA: orange and LPUFA: blue) diets contained 68\% of calories from carbohydrates, $19 \%$ from protein, and 13\% from fat. The high fat diets (HSFA: green and HPUFA: red) contained $43 \%$ of calories from carbohydrates, $15 \%$ from protein, and $42 \%$ from fat. c: An oral glucose tolerance test was performed in all animals, 4 and 8 weeks after start of the feeding protocol. Here, blood glucose levels of the 8 week time point are illustrated. Cumulative glucose as determined by area under the curve (AUC) in relative units (RU), is shown in the inset. d: Food consumption of all dietary groups was measured twice/week during the 10 week period. $\mathrm{n}=18-20$ animals per group. ${ }^{\mathrm{a}}, p<0.05$ compared to the according LFD group; ${ }^{\mathrm{b}}, p<0.05$ compared to HSFA 
a

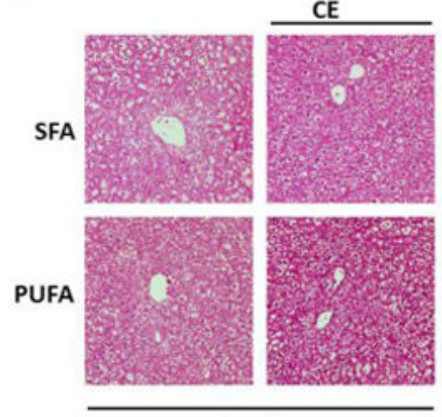

low fat

C

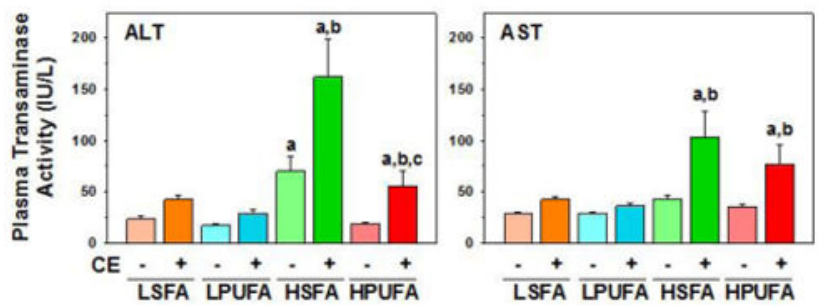

b

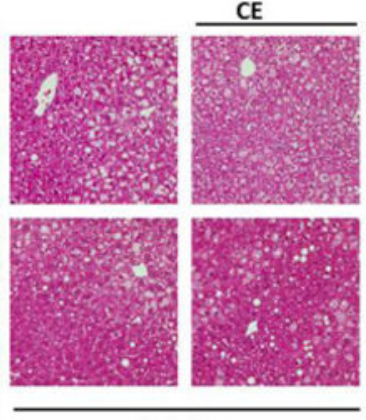

high fat

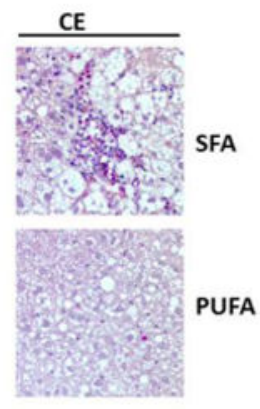

high fat

d

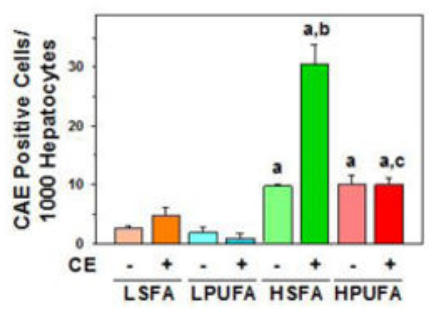

Figure 2. Differences in HFD-induced liver injury caused by CE in mice

a: Representative photomicrographs of H\&E stains (general pathology; 200x), and b: CAE (neutrophils, purple; 600×) stains are shown. c: ALT/AST were determined in plasma samples as described in Materials and Methods. d: CAE stain was quantitated by counting CAE positive cells as described in Materials and Methods, and were expressed as CAE positive cells per 1000 hepatocytes. $\mathrm{n}=8-10$ animals per group. ${ }^{\mathrm{a}}, p<0.05$ compared to the according LFD group; ${ }^{\mathrm{b}}, p<0.05$ compared to the absence of chloroethanol; ${ }^{\mathrm{c}}, p<0.05$ compared to HSFA 
a

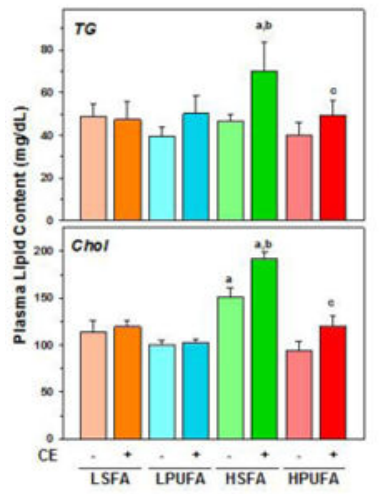

b

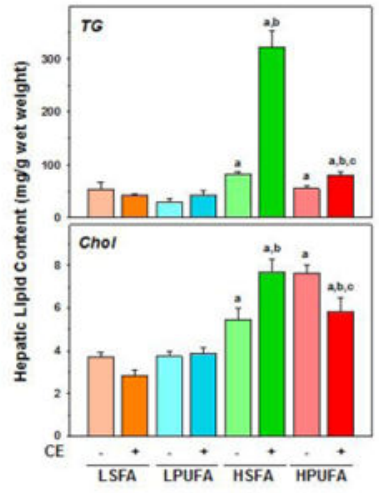

C

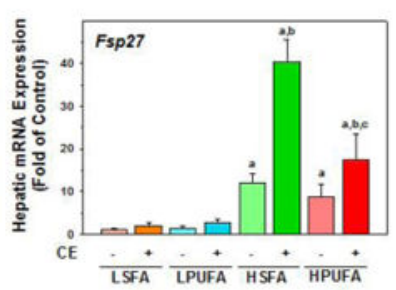

d

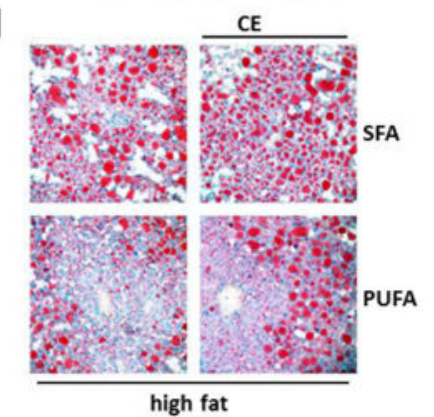

Figure 3. Changes in hepatic and circulating lipids in mice

Triglycerides and cholesterol were quantitatively measured in a: plasma (circulating lipids) and in b: hepatic lipid extracts. c: Hepatic mRNA expression of Fsp27 (lipid droplet formation) was determined by qRT-PCR. d: Representative photomicrographs of ORO stains for neutral lipids (red; 200x) are shown. $\mathrm{n}=8-10$ animals per group. ${ }^{\mathrm{a}}, p<0.05$ compared to the according LFD group; ${ }^{\mathrm{b}}, p<0.05$ compared to the absence of chloroethanol; ${ }^{\mathrm{c}}, p<0.05$ compared to HSFA 
a

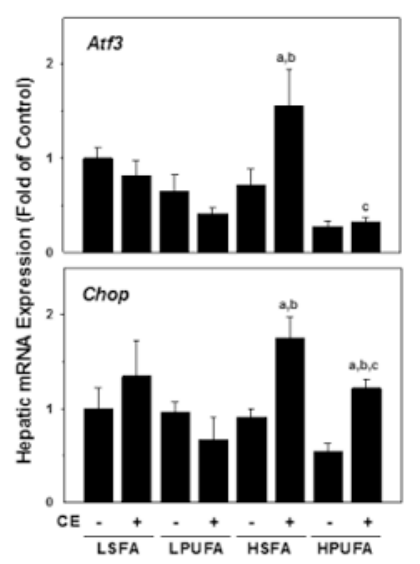

b

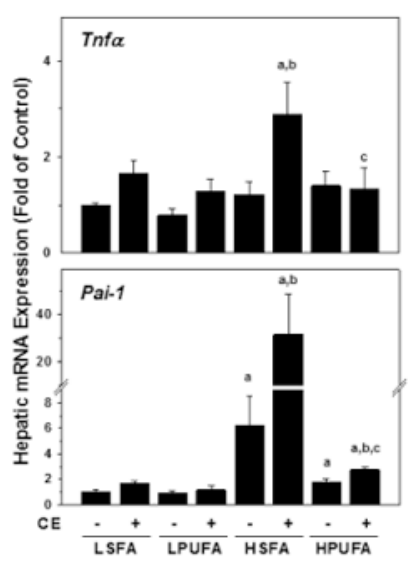

C

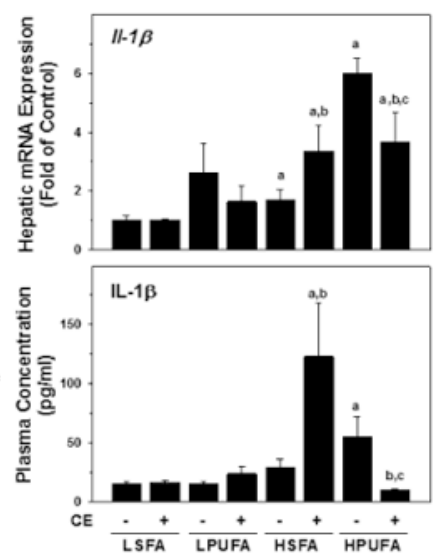

Figure 4. Differences in inflammatory and endoplasmic reticulum stress response caused by $\mathrm{CE}$ in mice fed saturated and unsaturated fatty diets

Real-Time RT-PCR was performed for genes involved in endoplasmic reticulum stress a: Atf3, Chop; and the inflammatory response b: Tnfa, Pai-1 and c: II-1 (upper panel). Plasma protein concentrations of IL-1 $\beta$ were measured via ELISA (lower panel). $n=8-10$ animals per group. ${ }^{\mathrm{a}}, p<0.05$ compared to the according LFD group; ${ }^{\mathrm{b}}, p<0.05$ compared to the absence of chloroethanol; ${ }^{\mathrm{c}}, p<0.05$ compared to HSFA 


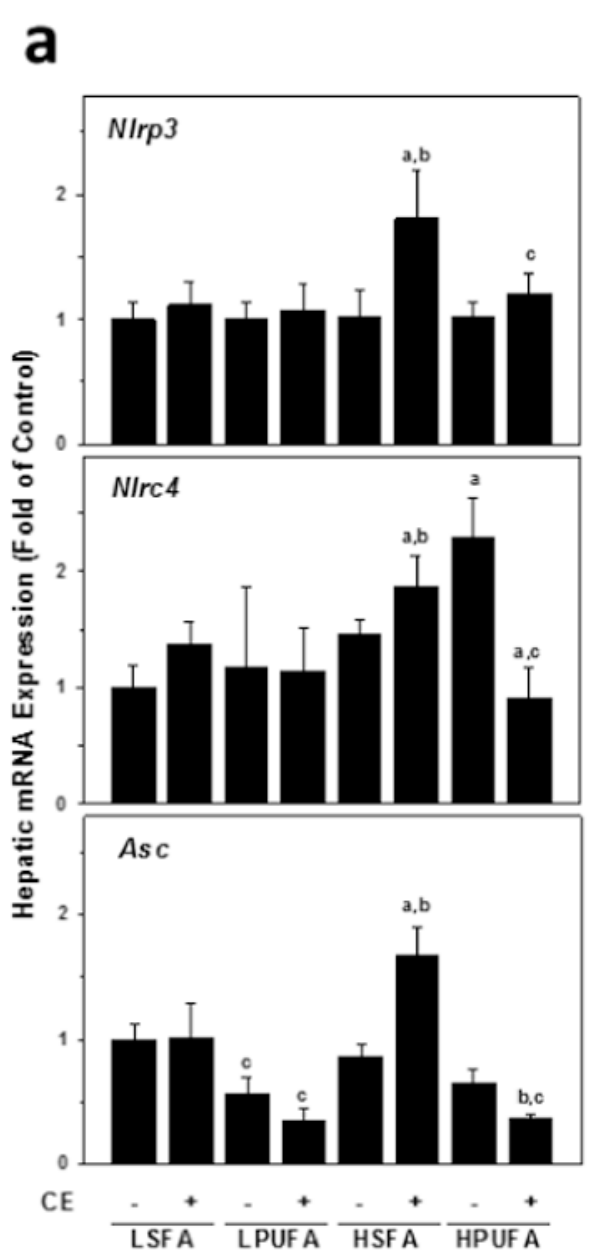

b
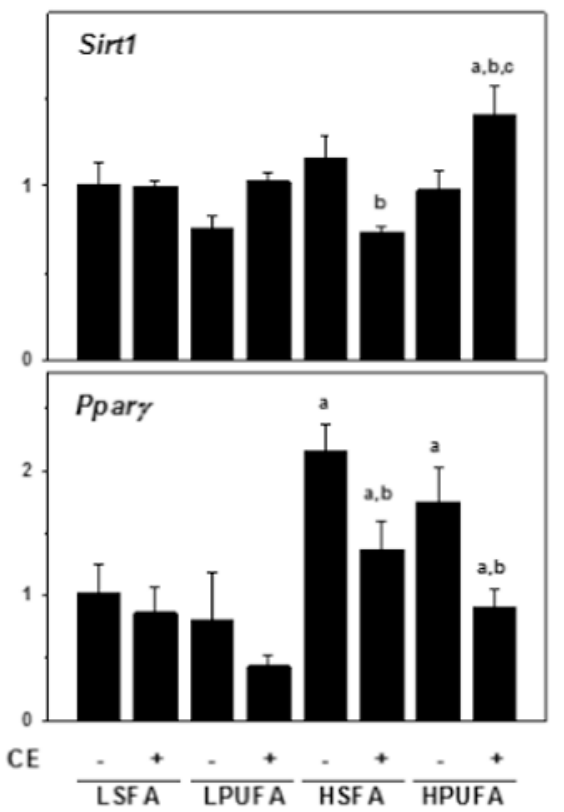

Figure 5. Differences in HFD-induced inflammasome activation caused by CE in mice Real-Time RT-PCR was performed for genes associated with a: inflammasome activation (NIrp3, NIrc4 and Asc), or b: inflammasome suppression (Sirt1 and Ppar $\gamma$ ). $\mathrm{n}=8-10$ animals per group. ${ }^{\mathrm{a}}, p<0.05$ compared to the according LFD group; ${ }^{\mathrm{b}}, p<0.05$ compared to the absence of chloroethanol; ${ }^{\mathrm{c}}, p<0.05$ compared to HSFA 

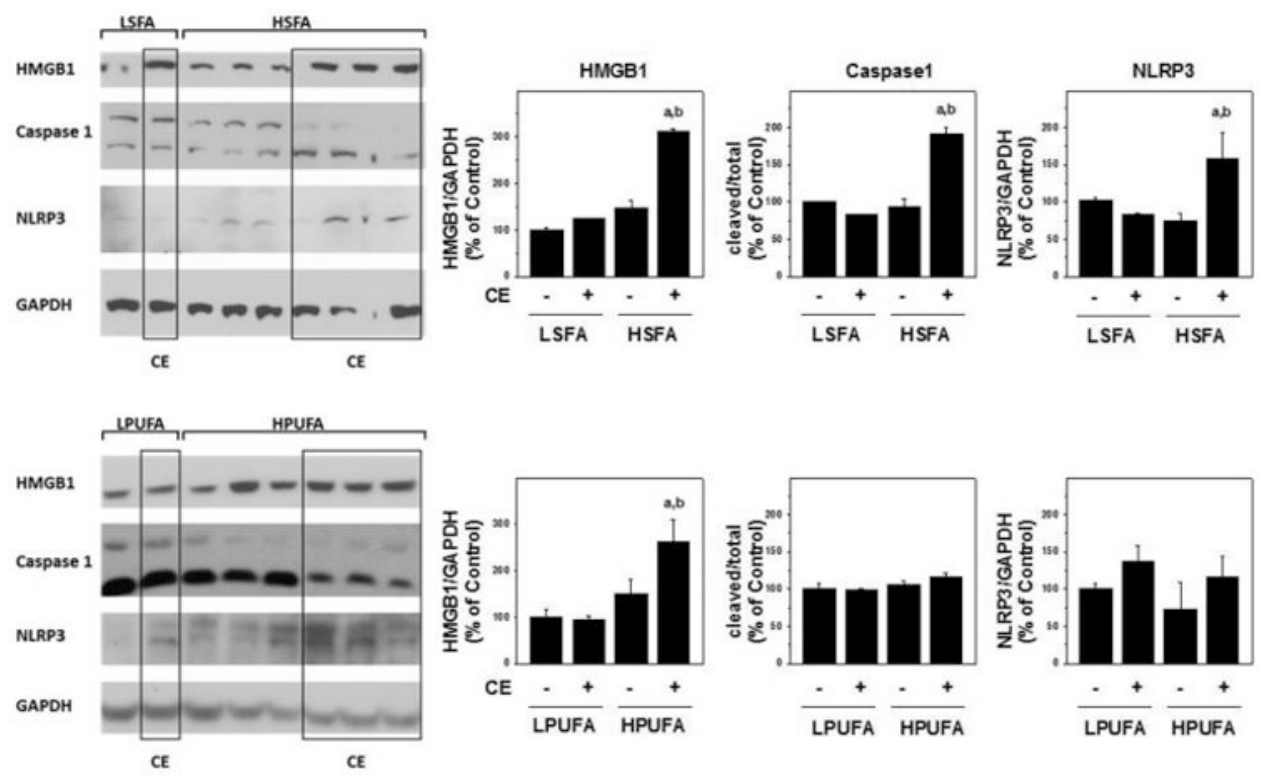

Figure 6. Differences in HFD-induced inflammasome activation caused by $\mathrm{CE}$ in mice Representative Western blots for HMGB1, Caspase1, and NLRP3 densitometric analyses of proteins are shown. $\mathrm{n}=8-10$ animals per group. ${ }^{\mathrm{a}}, p<0.05$ compared to the according LFD group; ${ }^{\mathrm{b}}, p<0.05$ compared to the absence of chloroethanol; ${ }^{\mathrm{c}}, p<0.05$ compared to HSF 